\title{
The Impact of Breast Cancer Resistance Protein (BCRP/ABCG2) on Drug Transport Across Caco-2 Cell Monolayers ${ }^{\text {[s }}$
}

\author{
lichiro Kawahara, ${ }^{1}$ Satoyo Nishikawa, ${ }^{2}$ Akira Yamamoto, Yusuke Kono, and Takuya Fujita \\ Department of Biopharmaceutics, Kyoto Pharmaceutical University, Kyoto, Japan (I.K., S.N., A.Y.) and Laboratory of Molecular \\ Pharmacokinetics, Graduate School of Pharmaceutical Sciences, Ritsumeikan University, Shiga, Japan (Y.K., T.F.)
}

Received July 10, 2019; accepted March 6, 2020

\begin{abstract}
Breast cancer resistance protein (BCRP) is expressed on the apical membrane of small intestinal epithelial cells and functions as an efflux pump with broad substrate recognition. Therefore, quantitative evaluation of the contribution of BCRP to the intestinal permeability of new chemical entities is very important in drug research and development. In this study, we assessed the BCRP-mediated efflux of several model drugs in Caco-2 cells using WK-X-34 as a dual inhibitor of P-glycoprotein (P-gp) and BCRP and LY335979 as a selective inhibitor of P-gp. The permeability of daidzein was high with an apparent permeability coefficient for apical-to-basal transport $\left(\boldsymbol{P}_{\mathrm{AB}}\right)$ of $20.3 \times 10^{-6} \mathrm{~cm} / \mathrm{s}$. In addition, its efflux ratio $(E R)$ was 1.55 , indicating that the contribution of BCRP to its transport is minimal. Estrone-3-sulfate and ciprofloxacin showed relatively higher ER values (>2.0), whereas their BCRP-related absorptive quotient $\left(A Q_{\mathrm{BCRP}}\right)$ was 0.21 and 0.3 , respectively. These results indicate that BCRP does not play a major role in regulating the permeability of estrone-3-sulfate and ciprofloxacin in Caco-2 cells. Nitrofurantoin showed a $P_{\mathrm{AB}}$ of $1.8 \times 10^{-6} \mathrm{~cm} / \mathrm{s}$, and its $E R$ was 7.6. However, the
\end{abstract}

\section{Introduction}

The oral route is the most desirable for drug administration because of its convenience, minimal invasiveness, and good patient compliance. The adequate bioavailability of drug candidates is required for them to be manufactured as orally available drugs. Intestinal permeability is an important factor that determines the bioavailability of drugs, and in many cases, it depends on the physicochemical properties of drugs, such as lipophilicity, molecular size, hydrogen bonding, and ionization (Jambhekar and Breen,

This study was supported by a Grant from the Strategic Research Foundation at Private Universities and Grant-in-Aids for Scientific Research (C) [17K08430] from the Ministry of Education, Culture, Sports, Science and Technology of Japan, the Ritsumeikan Global Innovation Research Organization (R-GIRO) Project at Ritsumeikan University.

${ }^{1}$ Current affiliation: Japan Tobacco Inc., Osaka, Japan.

${ }^{2}$ Current affiliation: Shionogi \& Co. Ltd., Osaka, Japan.

https://doi.org/10.1124/dmd.119.088674.

S This article has supplemental material available at dmd.aspetjournals.org.
$A Q_{\mathrm{BCRP}}$ was 0.37 , suggesting minimal contribution of $\mathrm{BCRP}$ to nitrofurantoin transport in Caco-2 cells. In contrast, topotecan, SN-38, and sulfasalazine had low $P_{A B}$ values $\left(0.81,1.13\right.$, and $0.19 \times 10^{-6} \mathrm{~cm} / \mathrm{s}$, respectively), and each $A Q_{\mathrm{BCRP}}$ was above 0.6 , indicating that $\mathrm{BCRP}$ significantly contributes to the transport of these compounds in Caco-2 cells. In conclusion, Caco-2 cells are useful to accurately estimate the contribution of BCRP to intestinal drug absorption.

\section{SIGNIFICANCE STATEMENT}

We performed an in vitro assessment of the contribution of breast cancer resistance protein (BCRP) to the transport of BCRP and/or P-glycoprotein (P-gp) substrates across Caco-2 cell monolayers using absorptive quotient, which has been proposed to represent the contribution of drug efflux transporters to the net efflux. The present study demonstrates that the combined use of a BCRP/P-gp dual inhibitor and a P-gp selective inhibitor is useful to estimate the impact of BCRP and P-gp on the permeability of tested compounds in Caco-2 cells.

ABBREVIATIONS: ABC, ATP-binding cassette; AP, apical; $A Q$, absorptive quotient; $\mathrm{BCRP}$, breast cancer resistance protein; $\mathrm{BL}$, basal; $\mathrm{DDI}$, drug-drug interaction; E3S, estrone-3-sulfate; $E R$, efflux ratio; GF120918, $N$-(4-(2-(6,7-dimethoxy-3,4-dihydroisoquinolin-2(1H)-yl)ethyl)phenyl)5-methoxy-9-oxo-9,10-dihydroacridine-4-carboxamide; HBSS, Hanks' balanced salt solution; LY335979, (R)-1-(4-((1aR,6s,10bS)-1,1-difluoro1,1a,6,10b-tetrahydrodibenzo[a,e]cyclopropa[c][7]annulen-6-yl)piperazin-1-yl)-3-(quinolin-5-yloxy)propan-2-ol trihydrochloride; MDCK, Madin-Darby canine kidney; MDR1, multidrug resistance protein 1; OATP, organic anion transporting polypeptide; P-gp, P-glycoprotein; PCR, polymerase chain reaction; WK-X-34, N-(2-\{4-[2-(6,7-dimethoxy-3,4-dihydro-1H-isochinolin-2-yl)-ethyl]phenylcarbamoyl\}phenyl)-3,4-dimethoxybenzamide. 
A

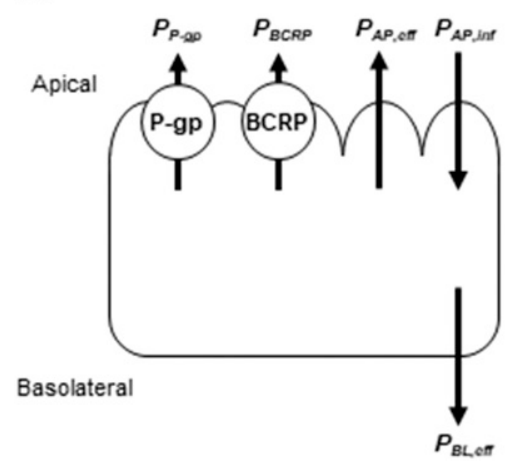

B

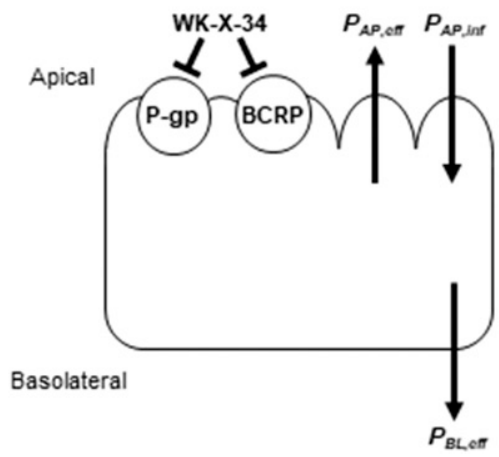

C

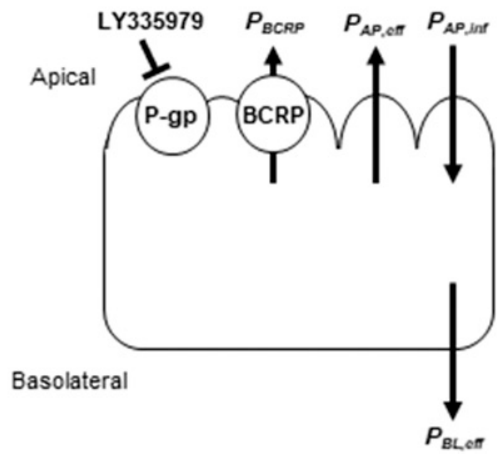

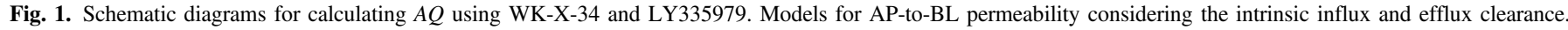

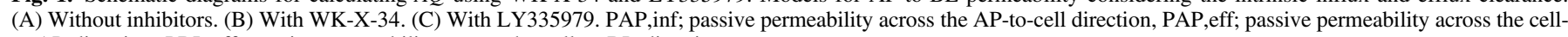
to-AP direction, PBL.eff; passive permeability across the cell-to-BL direction.

intestinal absorption of drugs using Caco-2 cell monolayers and mdr1a/ $1 \mathrm{~b}$ knockout mice (Fujita et al., unpublished data).

The BCRP expression level in human intestines has been reported to be equal to or even higher than that of multidrug resistance protein 1 (MDR1) (Doyle et al., 1998; Taipalensuu et al., 2001; Hilgendorf et al., 2007). BCRP is a member of the ATP-binding cassette (ABC) family of transporters. BCRP was discovered in multidrug-resistant cancer cells (Allikmets et al., 1998; Doyle et al., 1998; Miyake et al., 1999), but it is also expressed in normal cells, such as in the placenta, liver, brain, and intestine (Maliepaard et al., 2001). Because BCRP has one ATP-binding cassette and six transmembrane domains, it is known as a half-ABC transporter, which forms homodimers to create a functional transporter (Kage et al., 2002; Takada et al., 2005). BCRP and P-gp show broad substrate specificity for various endogenous substrates (bile acids, porphyrins, and folates), sulfate conjugates (estrone-3-sulfate), and anticancer drugs (mitoxantrone and topotecan) (Suzuki et al., 2003; Mao and Unadkat, 2005; Xiao et al., 2006). The role of BCRP in drug disposition in humans has been demonstrated in clinical studies. The oral bioavailability of topotecan in patients with cancer increased from 40 to $97 \%$ by coadministration of GF120918, a dual inhibitor of P-gp and BCRP (Kruijtzer et al., 2002), indicating that the role of BCRP in drug intestinal absorption cannot be ignored. The analysis of expression systems is useful to determine whether each drug is a substrate for BCRP, but it is difficult to evaluate the extent of its contribution to intestinal absorption. Therefore, it is necessary to quantitatively assess the impact of BCRP and P-gp on the intestinal absorption of drugs.

The Caco- 2 cell line is widely used as an in vitro intestinal model system to evaluate the intestinal permeability of new chemical entities. Importantly, Caco-2 cells exhibit many of the morphologic and functional properties of human intestinal epithelial cells. In addition to metabolic enzymes ((Fisher et al., 1999); (Siissalo et al., 2008)), they express many nutrient transport systems, such as various amino acid transporters ((Frølund et al., 2010); (Nielsen et al., 2012); (Voigt et al., 2013)), water-soluble vitamin transporters ((Wang et al., 1999); (Ramanathan et al., 2001)), and the $\mathrm{H}^{+}$-coupled di/tripeptide transporter ((Dantzig and Bergin, 1990); (Thwaites et al., 1994)). Various ABC transporters, such as P-gp and BCRP, are also expressed in Caco-2 cells (Taipalensuu et al., 2001; Volpe, 2008).

In the present study, we assessed the potential of efflux transporter inhibitors using paclitaxel and mitoxantrone. Among efflux transporter inhibitors, GF120918 and WK-X-34 are dual inhibitors of P-gp and BCRP, and LY335979 is a selective inhibitor of P-gp. Seven model compounds were selected to estimate the impact of BCRP in Caco-2 cells. The contribution of BCRP to the permeability of tested compounds across Caco- 2 cell monolayers, which express both ABC transporters, was assessed by using the subtraction of permeability method in the presence of a dual inhibitor or a P-gp selective inhibitor.

\section{Materials and Methods}

Chemicals and Reagents. Caffeine, Sepazol-RNA I Super, Dulbecco's modified Eagle's medium, antibiotic-antimycotic mixed stock solution $(100 \times)$, and $0.25 \%$ trypsin/ $1 \mathrm{mM}$ EDTA solution were purchased from Nacalai Tesque (Kyoto, Japan). Hanks' balanced salt solution (HBSS), daidzein, sulfasalazine, and $N$-(4-(2-(6,7-dimethoxy-3,4-dihydroisoquinolin-2(1H)-yl)ethyl)phenyl)-5-methoxy9-oxo-9,10-dihydroacridine-4-carboxamide (GF120918; elacridar) were obtained from Sigma-Aldrich (St. Louis, MO). SN-38 was purchased from Wako Pure Chemicals (Osaka, Japan). Ciprofloxacin was purchased from LKT Laboratories, Inc. (St. Paul, MN). Nitrofurantoin was purchased from MP Biomedicals, Inc.
A

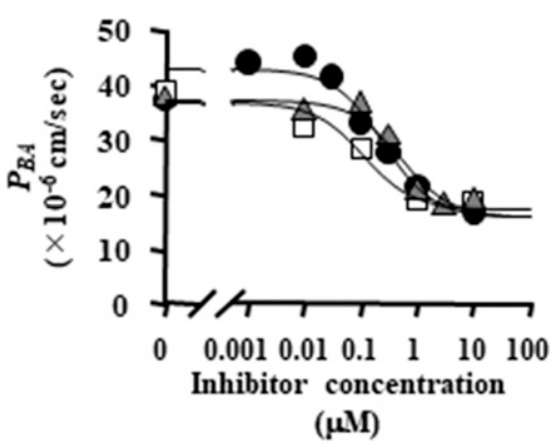

B

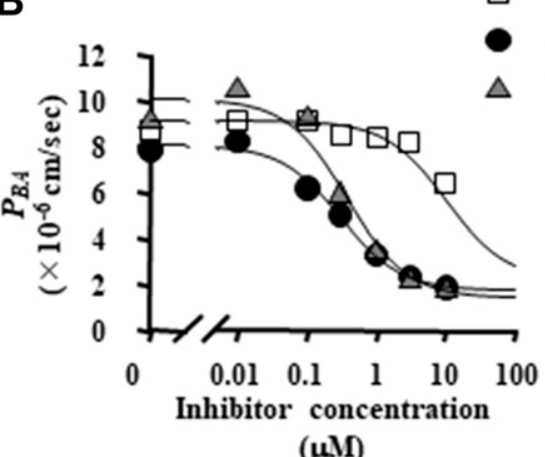

LY335979

GF120918

WK-X-34
Fig. 2. Inhibitory effects of LY335979, WK-X34, and GF120918 on P-gp and BCRP activity in Caco-2 cell monolayers. Concentration-dependent inhibitory effects of LY335979, WK-X-34, or GF120918 on the BL-to-AP transport of paclitaxel (A) and mitoxantrone (B) in Caco-2 cell monolayers. 


\section{A Caffeine}

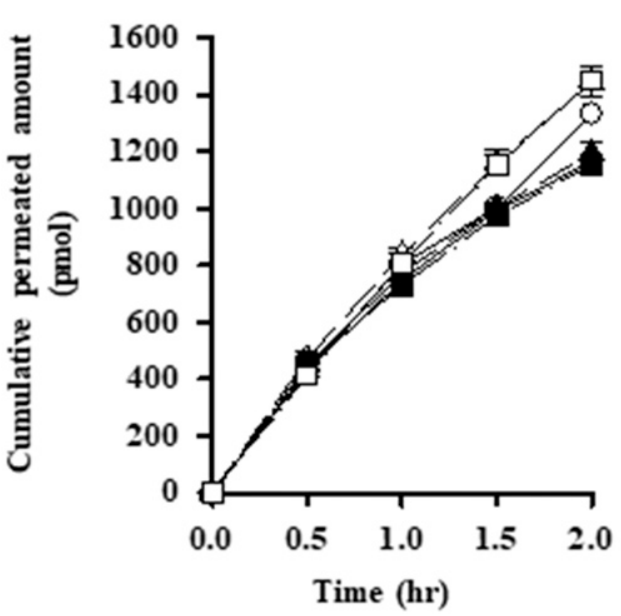

\section{B Mannitol}

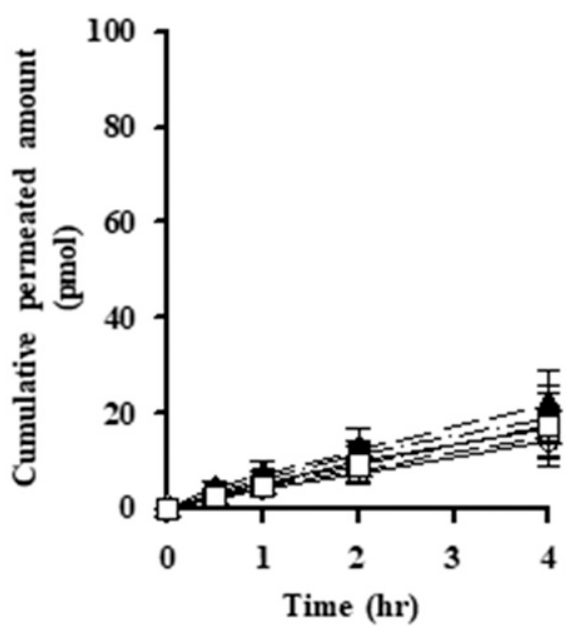

Fig. 3. Bidirectional transport of caffeine (A) and mannitol (B) across Caco-2 cell monolayers. The AP-to-BL and BL-to-AP transport of caffeine $(5 \mu \mathrm{M})$ and mannitol $(5 \mu \mathrm{M})$ in the absence or presence of WK-X-34 or LY335979 in Caco-2 cell monolayers. Data are represented as means \pm S.D. of three experiments. Keys: (O) control, A-to-B; $(\bullet)$ control, B-to-A; $(\mathbf{\Lambda})+$ WK-X-34, A-to-B; $(\triangle)+$ WK-X-34, B-to-A; (घ) + LY335979, A-to-B; ( $\square)$ + LY335979, B-to-A.
(Tokyo, Japan). Topotecan $\mathrm{HCl}$ was purchased from Alexis Corporation (Lausen, Switzerland). $N$-(2-\{4-[2-(6,7-Dimethoxy-3,4-dihydro- $1 H$-isochinolin-2-yl)ethyl]phenylcarbamoyl \}phenyl)-3,4-dimethoxybenzamide (WK-X-34) was purchased from Cosmo Bio Co. Ltd. (Tokyo, Japan). (R)-1-(4-((1aR,6s,10bS)-1,1difluoro-1,1a,6,10b-tetrahydrodibenzo[a,e]cyclopropa[c][7]annulen-6-yl)piperazin1-yl)-3-(quinolin-5-yloxy)propan-2-ol trihydrochloride (LY335979; zosuquidar) was purchased from Funakoshi (Tokyo, Japan). D-[1- $\left.{ }^{14} \mathrm{C}\right]$ Mannitol (specific radioactivity: $8 \mathrm{Ci} / \mathrm{mmol}$ ) was obtained from American Radiolabeled Chemicals Inc. (St. Louis, MO). $\left[6,7-{ }^{3} \mathrm{H}(N)\right]$ Estrone sulfate, ammonium salt (specific radioactivity: $57.3 \mathrm{Ci} / \mathrm{mmol}$ ) was purchased from PerkinElmer Life and Analytical Sciences (Waltham, MA). The Platinum Quantitative PCR SuperMix-UDG was purchased from Thermo Fisher Scientific K.K. (Kanagawa, Japan). Real-time polymerase chain reaction (PCR) primer sets were obtained from Eurofin Genomics (Louisville, KY). RT $(5 \times)$ buffer, dNTP mix, random primers, oligo $(\mathrm{dT})_{20}$, and ReverTra Ace were purchased from TOYOBO (Osaka, Japan). The Transwell membrane was purchased from Corning (Corning, NY). Other chemicals used were of the highest purity available.

Cell Culture. The human colon adenocarcinoma cell line Caco- 2 was obtained from DS Pharma Biomedical (Osaka, Japan). Caco-2 cells were maintained by serial passage in $75 \mathrm{~cm}^{2}$ plastic culture flasks. Caco-2 cells were routinely cultured with Dulbecco's modified Eagle's medium supplemented with $10 \%$ heatinactivated fetal bovine serum (Gibco Life Technologies, Waltham), $1 \%$ nonessential amino acids (Nacalai Tesque), and $100 \mathrm{U} / \mathrm{ml}$ penicillin $\mathrm{G}$ and $100 \mu \mathrm{g} / \mathrm{ml}$ streptomycin (Kono et al., 2017). Cells were passaged once they reached approximately $80 \%$ confluency using $0.02 \%$ EDTA and $0.05 \%$ trypsin. For transport experiments, Caco-2 cells were seeded on a polyethylene terephthalate insert $(0.4 \mu \mathrm{m}$ pore size, $12 \mathrm{~mm}$ diameter $)$ at a density of $1.0 \times 10^{5}$ cells per well and cultured for 3 weeks. The medium was replaced once every 3 days.

Real-Time Reverse Transcription PCR. Total RNA was isolated from Caco-2 cells after 3 weeks of culture using the Sepasol RNA I reagent according to the manufacturer's instructions. Gene-specific real-time PCR was performed using total RNA that was isolated from each cell culture using a master mix based on the Platinum Quantitative PCR SuperMix-UDG, primer pairs, and Taqman probes in a Taqman 7500 Sequence Detector according to the manufacturer's protocol. Data were normalized to the amount of glyceraldehyde-3-phosphate dehydrogenase mRNA in each sample. The primers used for real-time PCR are listed in Supplemental Table 1.

Transport Study. The confluent Caco-2 cells grown on Transwell membranes were washed with HBSS, followed by the addition of HBSS with or without the indicated concentration of inhibitors to both the apical (AP) and basal (BL) sides of the cell monolayer. After preincubation for 10 minutes, the experiment was initiated by replacing the HBSS at either the AP or BL side of the cell monolayer with HBSS containing each model compound and the corresponding inhibitor. The cells were incubated at $37^{\circ} \mathrm{C}$, and an aliquot of the medium was collected from each compartment at specified times. The amount of each model compound in the samples was measured. In addition, paracellular flux was monitored by the appearance of $\left[{ }^{14} \mathrm{C}\right]$ mannitol in the acceptor compartment.

Analytical Methods. To measure the radiolabeled compounds $\left(\left[{ }^{3} \mathrm{H}\right]\right.$ estrone sulfate and $\left[{ }^{14} \mathrm{C}\right]$ mannitol), the samples were transferred to counting vials, mixed with a scintillation cocktail (Clearsol I; Nacalai Tesque), and placed in a liquid scintillation counter (LS-6500; Beckman Instruments, Fullerton, CA). Other compounds were analyzed by a high-performance liquid chromatography system (Shimadzu LC-10AS pump, SIL-10A autosampler; Shimadzu) equipped with a reverse-phase column (COSMOSIL 5C 18 -AR-II, $3.5 \mu \mathrm{m}$ inner diameter, $4.6 \times$ $150 \mathrm{~mm}$ ). The flow rate was $1.0 \mathrm{ml} / \mathrm{min}$. The compositions of mobile phases were as follows: caffeine, $10 \mathrm{mM}$ phosphate buffer $(\mathrm{pH} 3.0)$ with methanol $(75: 25, \mathrm{v} / \mathrm{v})$; daidzein, $0.1 \%$ formic acid with acetonitrile $(73: 27, \mathrm{v} / \mathrm{v})$; nitrofurantoin, $10 \mathrm{mM}$ phosphate buffer $(\mathrm{pH} 3.0)$ with acetonitrile $(83: 17$, v/v); sulfasalazine, $5 \mathrm{mM}$ phosphate buffer ( $\mathrm{pH} 6.0)$ with acetonitrile $(78: 22, \mathrm{v} / \mathrm{v})$; ciprofloxacin, $10 \mathrm{mM}$ formate buffer $(\mathrm{pH} 3.0)$ with methanol and acetonitrile (82:9:9, v/v); SN-38, $10 \mathrm{mM}$ phosphate buffer ( $\mathrm{pH} 3.0)$ with acetonitrile (74:26, v/v); and topotecan, $10 \mathrm{mM}$ phosphate buffer ( $\mathrm{pH} 3.7)$ with methanol (76:24, v/v). Caffeine, daidzein, nitrofurantoin, and sulfasalazine were detected by absorbance at 273, 250, 366, and $357 \mathrm{~nm}$, respectively, using a Shimadzu SPD-20A UV spectrophotometric detector. Ciprofloxacin (excitation: $280 \mathrm{~nm}$, emission: $460 \mathrm{~nm}$ ), SN-38 (excitation: $380 \mathrm{~nm}$, emission: $550 \mathrm{~nm}$ ), and topotecan (excitation: $361 \mathrm{~nm}$, emission:

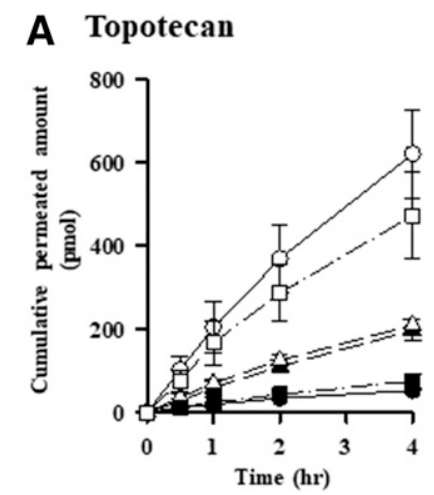

B Estrone-3-sulfate

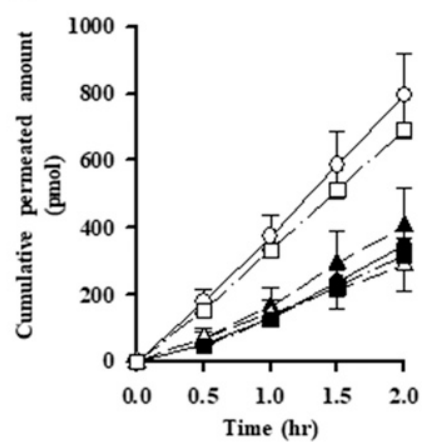

Fig. 4. Bidirectional transport of topotecan (A) and estrone-3-sulfate (B) across Caco- 2 cell monolayers. The AP-to-BL and BL-to-AP transport of topotecan $(5 \mu \mathrm{M})$ and estrone-3-sulfate $(5 \mu \mathrm{M})$ in the absence or presence of WK-X-34 or LY335979 in Caco-2 cell monolayers. Data are represented as means \pm S.D. for three experiments. Keys: $(\bigcirc)$ control, A-to-B; $(\bigcirc)$ control, B-to-A; $(\mathbf{A})+$ WK-X-34, A-to-B; $(\triangle)+$ WK-X-34, B-to-A; $(\mathbf{\square})+$ LY335979, A-to-B; $(\square)+$ LY335979, B-to-A. 
$527 \mathrm{~nm}$ ) were analyzed by measuring fluorescence with a Shimadzu RF-10A XL fluorescence detector.

Data Analysis. The apparent permeability coefficient $(P)$ was calculated using eq. 1 ,

$$
P=\Delta Q / \Delta t \times 1 / \mathrm{A} \cdot C_{0}
$$

where $\Delta Q / \Delta t$ is the transported flux of a compound, A is the surface area of the porous membrane $\left(1.13 \mathrm{~cm}^{2}\right)$, and $C_{0}$ is the initial concentration of a test compound added to the donor compartment.

The inhibitory potency for BCRP was determined for each inhibitor. The following equation (eq. 2) was fitted to the collected permeability data:

$$
P_{\mathrm{BA}}=\text { Range } /\left[1+\left(C / I C_{50}\right)^{\gamma}\right]+\text { Background }
$$

where $P_{\mathrm{BA}}$ is the $P$ value for BL-to-AP transport, $C$ is the concentration of an inhibitor, $\gamma$ is the Hill coefficient, Range is the arithmetic difference of the $P_{\mathrm{BA}}$ value between complete inhibition and in the absence of inhibitors, Background is the $P_{\mathrm{BA}}$ in the absence of inhibitors, and $I C_{50}$ is the inhibitor concentration that achieves $50 \%$ inhibition.

The efflux ratio $(E R)$, a parameter that is widely used as an index of efflux transporter activity, was calculated using eq. 3 :

$$
E R=P_{\mathrm{BA}} / P_{\mathrm{AB}}
$$

where $P_{\mathrm{AB}}$ is the $P$ value for AP-to-BL transport.

Absorptive quotient $(A Q)$ has been proposed as an experimental parameter to quantify the contribution of $\mathrm{P}-\mathrm{gp}$ to intestinal absorption (Troutman and Thakker, 2003). $A Q$ was calculated according to the following equation:

$$
A Q=\left(P_{\mathrm{AB},+ \text { inhibitor }}-P_{\mathrm{AB}}\right) / P_{\mathrm{AB},+ \text { inhibitor }}
$$

where $P_{\mathrm{AB},+ \text { inhibitor }}$ is the $P_{\mathrm{AB}}$ determined in the presence of an inhibitor.

Based on the above theory and the report of Tachibana et al. (2010), we determined the $A Q$, which was recently proposed to represent the contribution of efflux transporters to the net efflux, using eqs. 5-7,

$$
\begin{aligned}
A Q_{\text {efflux }} & =\frac{P_{A B,+W K-X-34}-P_{A B}}{P_{A B,+W K}-X-34}=\frac{P_{P-g p}+P_{B C R P}}{P_{A P, e f f}+P_{B L, e f f}+P_{P-g p}+P_{B C R P}} \\
A Q_{P-g p} & =\frac{P_{A B,+L Y 335979}-P_{A B}}{P_{A B,+W K-X-34}} \\
& =\frac{\left(P_{A P, e f f}+P_{B L, e f f}\right) \cdot P_{P-g p}}{\left(P_{A P, e f f}+P_{B L, e f f}+P_{P-g p}+P_{B C R P}\right) \cdot\left(P_{A P, e f f}+P_{B L, e f f}+P_{B C R P}\right)} \\
A Q_{B C R P} & =\frac{P_{A B,+W K-X-34}-P_{A B,+L Y 335979}}{P_{A B,+W K-X-34}}=\frac{P_{B C R P}}{P_{A P, e f f}+P_{B L, e f f}+P_{B C R P}}
\end{aligned}
$$

where $A Q_{\text {efflux }}$ is both P-gp- and BCRP-related $A Q, A Q_{P-g p}$ is P-gp-related $A Q$, $A Q_{B C R P}$ is BCRP-related $A Q, P_{A P}$, eff is the passive permeability across the AP membrane in a cellular-to-AP direction, $P_{B L \text {, eff }}$ is the passive permeability across the BL membrane in a cellular-to-BL direction, $P_{\mathrm{p}-\mathrm{gp}}$ is the permeability due to P-gp-mediated efflux activity, and $P_{B C R P}$ is the permeability due to BCRPmediated efflux activity (Fig. 1).

Statistical Analysis. Analysis of variance was used to test the statistical significance of differences between groups. Multiple comparisons between control and other groups were performed with Dunnett's test.

\section{Results}

Effects of Inhibitors on the BL-to-AP Transport of P-gp and BCRP Substrates. We evaluated the inhibitory effect of GF120918, WK-X-34, and LY335979 on the BL-to-AP transport of paclitaxel (P-gp substrate) and mitoxantrone (BCRP substrate) across Caco-2 cell monolayers to identify the most potent inhibitor of P-gp and BCRP. Prior to transport studies, we determined the gene expression of $M D R 1$, $B C R P$, and Multidrug Resistance-associated Protein 2 (MRP2) in Caco2 cells, and the expression level of $B C R P$ was higher than that of $M D R 1$ (data not shown). This gene expression pattern in Caco-2 cells was
A Daidzein

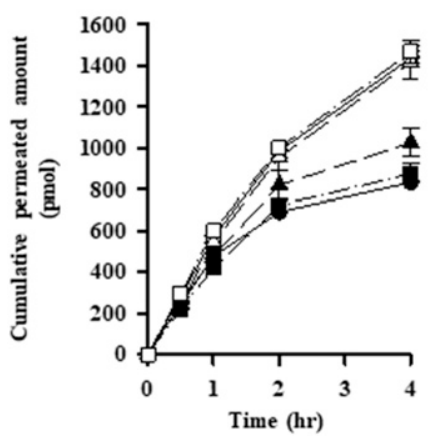

B Ciprofloxacin

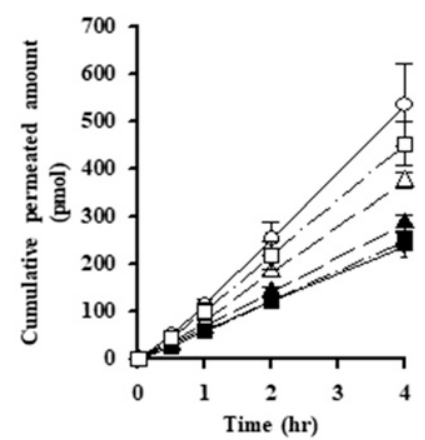

Fig. 5. Bidirectional transport of daidzein (A) and ciprofloxacin (B) across Caco-2 cell monolayers. The AP-to-BL and BL-to-AP transport of daidzein $(5 \mu \mathrm{M})$ and ciprofloxacin $(5 \mu \mathrm{M})$ in the absence or presence of WK-X-34 or LY335979 in Caco2 cell monolayers. Data are represented as means \pm S.D. for three experiments. Keys: (○) control, A-to-B; $(\bigcirc)$ control, B-to-A; $(\mathbf{\Delta})+$ WK-X-34, A-to-B; $(\triangle)+$ WK-X-34, B-to-A; (ם) + LY335979, A-to-B; $(\square)+$ LY335979, B-to-A.

comparable to what is observed in the human small intestine. The cytochrome P450 $3 A 4$ gene was also expressed in Caco-2 cell monolayers, although the expression level was not as high as that in human intestines. As shown in Fig. 2, the transport of paclitaxel and mitoxantrone was inhibited by GF120918 in a concentration-dependent manner with $I C_{50}$ values of 239 and $298 \mathrm{nM}$, respectively. In addition, the respective $I C_{50}$ values of $\mathrm{WK}-\mathrm{X}-34$ were 501 and $370 \mathrm{nM}$, and thus both GF120918 and WK-X-34 were identified as a dual inhibitor of P-gp and BCRP. In particular, the $I C_{50}$ value of $\mathrm{WK}-\mathrm{X}-34$ for mitoxantrone transport was lower than that for paclitaxel. In addition, it has been reported that WK-X-34 has no inhibitory effect on MRP2-mediated transport of 5-carboxyfluorescein diacetate in MRP2-expressing MadinDarby canine kidney (MDCK) cells (Jekerle et al., 2006, 2007). Taking these findings into consideration, WK-X-34 inhibits BCRP more selectively than P-gp and MRP2. In contrast, the $I C_{50}$ value of LY33579 for paclitaxel was $107 \mathrm{nM}$, whereas its inhibitory effect on mitoxantrone transport was minimal $\left(I C_{50}>10 \mu \mathrm{M}\right)$. Therefore, these results indicate that LY335979 is a selective inhibitor of P-gp. Based on these results, WK-X-34 at $10 \mu \mathrm{M}$ was used as an inhibitor of both BCRP and P-gp, and LY335979 at $1 \mu \mathrm{M}$ was used as a selective P-gp inhibitor.

Assessment of the Contribution of BCRP to the Transport of BCRP Substrates Across Caco-2 Cell Monolayers. To determine the contribution of BCRP and P-gp to the permeability of drugs across Caco- 2 cell monolayers, we conducted a transport study in the presence of WK-X-34 and LY335979. Initially, we assessed the effect of WK-X34 and LY335979 on the transport of caffeine and mannitol, which permeate only by passive diffusion. The $P_{\mathrm{AB}}$ and $P_{\mathrm{BA}}$ values of both caffeine and mannitol did not change in the presence of WK-X-34 and LY335979 (Fig. 3). Next, we investigated the transport of topotecan and estrone-3-sulfate (E3S), which are typical substrates of BCRP, across Caco- 2 cell monolayers. Topotecan exhibited an ER of 12.7, indicating the involvement of efflux transporters in its transport (Fig. 4A). Moreover, the transport of topotecan was not inhibited in the presence of LY335979, whereas its AP-to-BL transport was increased, and its BL-to-AP transport was decreased in the presence of WK-X-34. These results indicate that topotecan is a favored BCRP-specific substrate. Furthermore, E3S exhibited an ER of 2.1 (Fig. 4B), suggesting the involvement of efflux transporters. However, its absorptive permeability was relatively high $\left(P_{\mathrm{AB}}=9.88 \times 10^{-6} \mathrm{~cm} / \mathrm{s}\right)$, which was not changed by the addition of WK-X-34. These results suggest that the contribution of BCRP to the absorptive transport of E3S is low. In contrast, the BL-toAP permeability of E3S was significantly decreased in the presence of 


\section{A Nitrofurantoin}

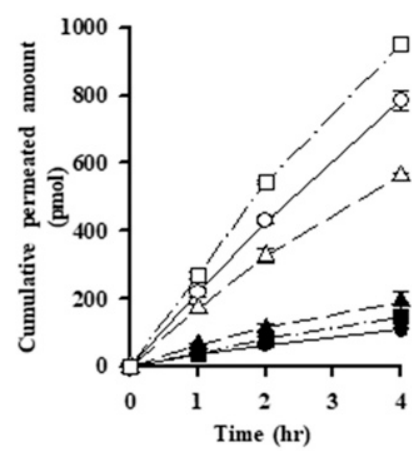

B $\quad \mathbf{S N}-38$

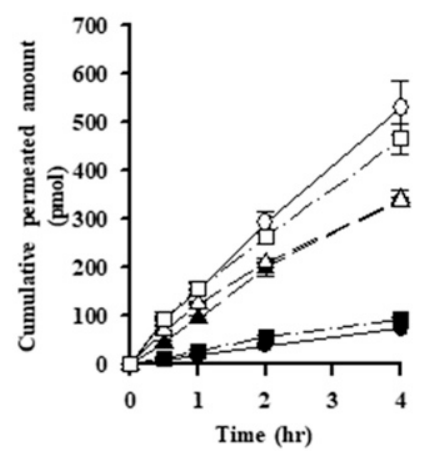

C Sulfasalazine

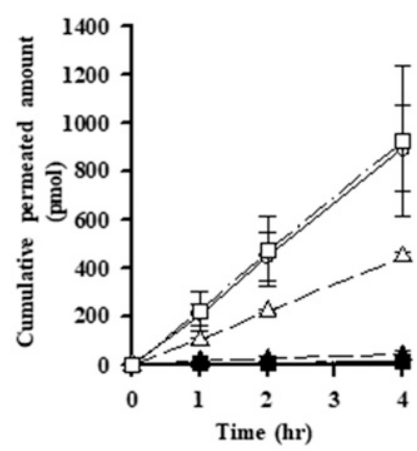

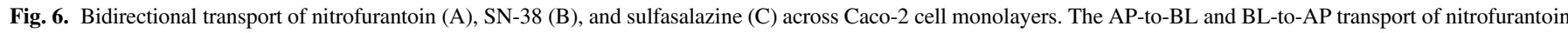

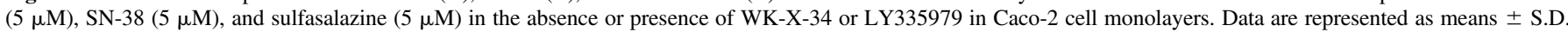

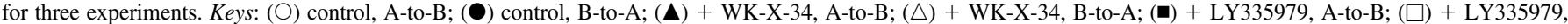
B-to-A.

WK-X-34, indicating that BCRP plays a role in the secretory transport of E3S in Caco-2 cells.

Assessment of the Contribution of BCRP to the Transport of Model Drugs Across Caco-2 Cell Monolayers. We investigated the contribution of BCRP to the permeability of daidzein, ciprofloxacin, nitrofurantoin, SN-38, and sulfasalazine in Caco-2 cells. The absorptive permeability of daidzein was high $\left(P_{\mathrm{AB}}=20.5 \times 10^{-6} \mathrm{~cm} / \mathrm{s}\right)$ and saturable (Fig. 5A). Moreover, its $E R$ was 1.5, and the effect of inhibitors on daidzein transport was not observed. These data suggest that the contribution of BCRP to daizdein transport is minimal in Caco-2 cell monolayers. The $E R$ of ciprofloxacin was 2.3 (Fig. 5B), suggesting the involvement of efflux transporters in its transport. However, the calculated $A Q_{\text {efflux }}$ was 0.15 . These results show that P-gp and BCRP do not play a major role in the transport of ciprofloxacin. In contrast, nitrofurantoin exhibited an $E R$ of 7.6, and its $A Q_{\text {efflux }}$ was 0.46 (Fig. 6A). Therefore, these data indicate the contribution of efflux transporters to the transport of nitrofurantoin in Caco-2 cell monolayers. We also determined that the $A Q_{\mathrm{BCRP}}$ and $A Q_{\mathrm{P} \text {-gp }}$ of nitrofurantoin were 0.37 and 0.09 , respectively. This indicates that $\mathrm{P}-\mathrm{gp}$ hardly contributes to nitrofurantoin transport. In the case of SN-38 and sulfasalazine, their $E R$ s were 6.73 and 71.6, respectively (Fig. 6, B and C). This indicates that the transport of both drugs, particularly sulfasalazine, is mediated by efflux transporters. Moreover, the Caco-2 permeability of SN-38 and sulfasalazine were not changed in the presence of LY33579 $\left(A Q_{\mathrm{P}-\mathrm{gp}}=\right.$ 0 and 0.10 , respectively), indicating that their transport is not mediated by P-gp. However, the transport of SN-38 and sulfasalazine were significantly increased in the presence of $\mathrm{WK}-\mathrm{X}-34$, and their $A Q_{\mathrm{P}-\mathrm{gp}}$ values were 0.74 and 0.59 , respectively. These results demonstrate that BCRP greatly affects the permeability of SN-38 and sulfasalazine across Caco-2 monolayers.

\section{Discussion}

In the present study, we investigated the BCRP-mediated efflux of nine compounds with various physicochemical properties in Caco-2 cells to determine the contribution of BCRP to the intestinal absorption of drugs.

Using this Caco-2 cell monolayer, we showed that the permeability of daidzein in the absorptive direction was quite high $\left(P_{\mathrm{AB}}=20.3 \times 10^{-6}\right.$ $\mathrm{cm} / \mathrm{s})$, and it was slightly increased in the presence of WK-X-34 $\left(P_{\mathrm{AB},+ \text { WK-X-34 }}=29.6 \times 10^{-6} \mathrm{~cm} / \mathrm{s}\right)($ Fig. $5 \mathrm{~A}$; Table 1$)$. Kobayashi et al. (2013) reported that the AP-to-BL transport of daidzein across Caco-2 cells was increased by approximately 1.7-fold in the presence of ES3 as a BCRP substrate. This result is consistent with the present observation. These findings suggest a minimal contribution of BCRP to the transport of daidzein in Caco- 2 cells. The relatively low $A Q_{\mathrm{BCRP}}$ and $E R$ values of daidzein (0.36 and 1.55) also support our findings. However, Enokizono et al. (2007) demonstrated that the area under the curve value of daidzein after its oral administration was 3.7-fold higher in $\operatorname{Bcrp}(-/-)$ mice compared with wild-type mice. Similar results were reported by(Álvarez et al., 2011) and (Ge et al., 2017). These reports indicated that BCRP is involved in the intestinal absorption of daidzein in mice. However, the area under the curve of daidzein after intravenous administration was also increased in $\mathrm{Bcrp}(-/-)$ mice. Taking these studies into consideration, we suggest that BCRP is responsible for the elimination of daidzein from the systemic circulation rather than its intestinal absorption.

Our results suggested that the transport of E3S and ciprofloxacin also involved efflux transporters because their $E R$ values were above 2.0 (Figs. $4 \mathrm{~B}$ and $5 \mathrm{~B}$; Table 1 ). The $A Q_{\mathrm{BCRP}}$ value of E3S was 0.21 , indicating that BCRP slightly contributes to its permeability across Caco- 2 cells. Gram et al. (2009) showed that the $P_{\mathrm{AB}}$ value of E3S in Caco- 2 cells increased by 1.4-fold in the presence of fumitremorgin $\mathrm{C}$, a BCRP inhibitor. This finding is similar to our present result. Sai et al. (2006) demonstrated that the uptake of E3S into Caco-2 cells was mediated by organic anion transporting polypeptide (OATP) 2B1 expressed in the apical membrane. It has also been reported that E3S transported into cells by OATP2B1 is exported to the outside of cells by BCRP (Grube et al., 2007). In the present study, since the permeability of E3S in the absorptive direction was relatively high $\left(P_{\mathrm{AB}}=9.88 \times\right.$ $\left.10^{-6} \mathrm{~cm} / \mathrm{s}\right)$ and its permeability in the absorptive direction was superior to that in the secretory direction in the presence of WK-X-34 $\left(P_{\mathrm{AB},+\mathrm{WK}-}\right.$ $\left.\mathrm{X}-34=11.3, P_{\mathrm{BA},+\mathrm{WK}-\mathrm{X}-34}=7.31 \times 10^{-6} \mathrm{~cm} / \mathrm{s}\right)$, it is assumed that OATP2B1 and BCRP functionally interact during the transport of E3S in Caco- 2 cells. The $A Q_{\mathrm{BCRP}}$ value of ciprofloxacin was 0.03 , suggesting that BCRP is not a major transporter of ciprofloxacin in Caco- 2 cells. Merino et al. (2006) demonstrated that Ko143, a potent and specific BCRP inhibitor, did not affect the AP-to-BL transport of ciprofloxacin in BCRP-expressing MDCKII cells. This observation supports our present result. However, they have also shown that Ko143 decreased the BL-toAP transport of ciprofloxacin. Since we observed that the permeability of ciprofloxacin in the secretory direction in the presence of WK-X-34 was lower than that in the presence of LY335979 $\left(P_{\mathrm{BA},+\mathrm{WK}-\mathrm{X}-34}=\right.$ $\left.4.44 \times 10^{-6} \mathrm{~cm} / \mathrm{s}, P_{\mathrm{BA},+\mathrm{LY} 335979}=5.80 \times 10^{-6} \mathrm{~cm} / \mathrm{s}\right)$, the present results are consistent with the observations reported by Merino et al. In addition, our findings are also in line with the report of Haslam et al. 


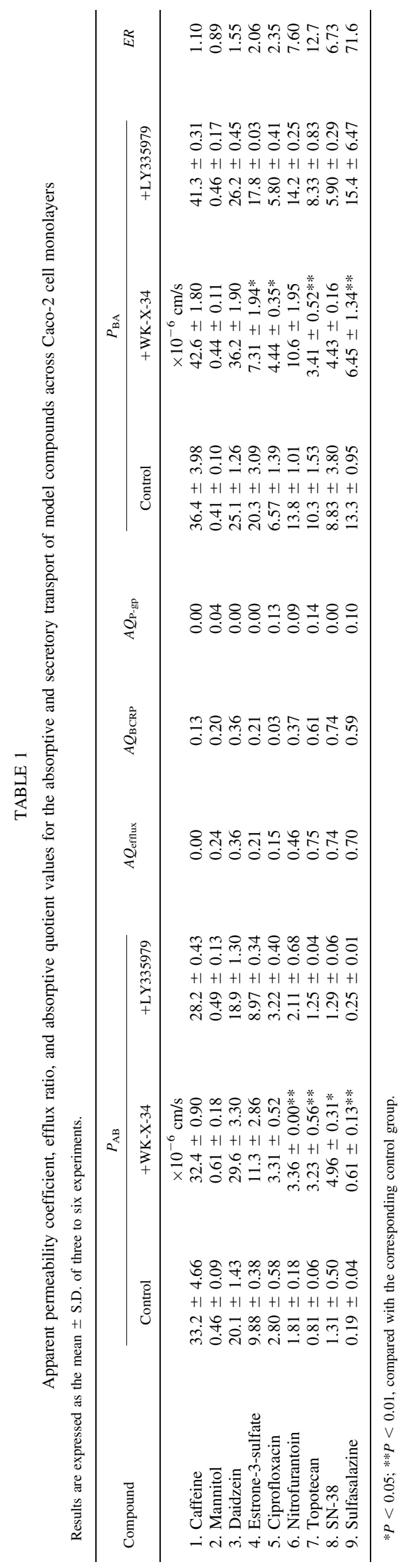

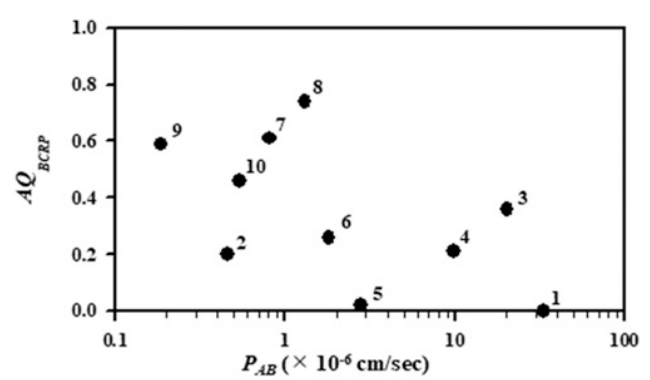

Fig. 7. The relationship between $P_{A B}$ and $A Q_{\mathrm{BCRP}}$ of selected compounds. Key: 1 , caffeine; 2, mannitol; 3, daidzein; 4, estrone-3-sulfate; 5, ciprofloxacin; 6, nitrofurantoin; 7, topotecan; $8, \mathrm{SN}-38 ; 9$, sulfasalazine; 10 , mitoxantrone.

(2011), which showed that Ko143 reduced the secretory net flux of ciprofloxacin in high-passage Caco-2 cells (115-120 passages) but had no significant impact in low-passage Caco-2 cells (34-40 passages). In addition, ciprofloxacin has also been reported to be a substrate for influx transporters, including OATP1A2 (Dautrey et al., 1999; Maeda et al., 2007; $\mathrm{Lu}$ et al., 2018). Moreover, its fraction absorbed in human intestines was estimated to be $70-100 \%$ (Drusano et al., 1986). In addition, the plasma concentrations of ciprofloxacin after oral and intravenous injection in wild-type mice were 2-fold and 1.5-fold higher than that in $\mathrm{Bcrp}(-/-)$ mice, respectively. Taking these findings into consideration, the contribution of BCRP would be higher in the elimination process of ciprofloxacin compared with its intestinal absorption.

Nitrofurantoin showed $P_{\mathrm{AB}}$ and $E R$ values of $1.8 \times 10^{-6} \mathrm{~cm} / \mathrm{s}$ and 7.6, respectively, suggesting the involvement of efflux transporters (Fig. 6A; Table 1). However, its $A Q_{\mathrm{BCRP}}$ was relatively low $(0.37)$. Moreover, the $E R$ value of nitrofurantoin was above 3, even in the presence of WK-X-34. It has been reported that the transepithelial secretion of nitrofurantoin in Caco-2 cells was inhibited by $92 \%$ in the presence of Ko143 due to a decrease in BL-to-AP flux and an increase in AP-to-BL flux (Wright et al., 2011). Similar results were obtained in BCRP-expressing MDCKII cells (Merino et al., 2005). Therefore, we suggest that WK-X-34 has a low affinity toward nitrofurantoin. In summary, it remains unclear whether BCRP acts as a barrier for nitrofurantoin permeability in Caco- 2 cells.

The permeability of topotecan, SN-38, and sulfasalazine in the absorptive direction was lower than other compounds $\left(P_{\mathrm{AB}}=0.81\right.$, 1.31 , and $0.19 \times 10^{-6} \mathrm{~cm} / \mathrm{s}$, respectively) (Figs. 4A and 6, B and C; Table 1). Moreover, each $A Q_{\mathrm{BCRP}}$ value was above 0.6 . These results demonstrate that BCRP significantly contributes to the transport of these drugs in Caco-2 cell monolayers. Matsuda et al. (2013) demonstrated that the intestinal availability of topotecan and sulfasalazine after oral administration in rats was increased by approximately 4-fold after the preadministration of Ko143. Moreover, Dahan and Amidon (2009) showed that the BCRP inhibitors fumitremorgin $\mathrm{C}$ and pantoprazole significantly increased the AP-to-BL transport of sulfasalazine. Our present results are in agreement with these findings. In addition, they also demonstrated that BCRP inhibitors decrease the BL-to-AP transport of sulfasalazine in Caco-2 cells. We observed that the permeability of sulfasalazine in the secretory direction in the presence of WK-X-34 was much lower than that in the presence of LY335979 $\left(P_{\mathrm{BA},+ \text { WK-X-34 }}=\right.$ $\left.6.45, P_{\mathrm{BA},+\mathrm{LY} 335979}=15.4 \times 10^{-6} \mathrm{~cm} / \mathrm{s}\right)$, and therefore, our results are consistent with Dahan's previous observation. With respect to sulfasalazine, it has been reported that Caco- 2 cells express OATP2B1 and that sulfasalazine is a substrate of OATP2B1 (Sai et al., 2006; Kusuhara et al., 2012). Therefore, sulfasalazine would be taken up from the AP side of the Caco- 2 cell monolayer by OATP2B1, although the membrane 
permeability of sulfasalazine is low because of its hydrophilicity. In addition, we also confirmed that the intracellular amount of sulfasalazine when it was added to the BL side of the Caco- 2 cell monolayer was much higher than when it was added to the AP side $(14.0 \mathrm{nmol} / \mathrm{mg}$ protein vs. $6.62 \mathrm{nmol} / \mathrm{mg}$ protein). This result suggests that the BL uptake of sulfasalazine may also involve transporters.

In contrast, the $A Q_{\mathrm{P}-\mathrm{gp}}$ values of topotecan, $\mathrm{SN}-38$, and sulfasalazine were less than 0.1 . This result suggests that $\mathrm{P}$-gp is not a major contributor to their permeability in Caco-2 cell monolayers. However, according to eq. 7 , the $A Q_{\mathrm{P}-\mathrm{gp}}$ was actually determined by the following equation when the transport studies were performed in the presence of a BCRP selective inhibitor, such as Ko143:

$$
A Q_{P-g p}=\frac{P_{P-g p}}{P_{A P, e f f}+P_{B L, e f f}+P_{P-g p}} .
$$

Since the $A Q_{\mathrm{P} \text {-gp }}$ determined by eq. 6 included $P_{\mathrm{BCRP}}$ in the denominator, the $A Q_{\mathrm{P} \text {-gp }}$ values calculated in this study were likely underestimated. Although the drugs used in this study were BCRP-specific substrates, and therefore, the underestimation of the $A Q_{\mathrm{P}-\mathrm{gp}}$ values would not be highly significant, it is necessary to consider this when determining the $A Q_{\mathrm{P}-\mathrm{gp}}$ of drugs that are substrates for both P-gp and BCRP.

To date, several BCRP selective inhibitors have been developed and used for investigating the influence of BCRP on drug permeation across intestinal epithelial cells in vitro. Particularly, Ko143 is a widely used and valuable inhibitor for determining the contribution of BCRP to drug transport in Caco- 2 cells. However, our method, together with the use of BCRP selective inhibitors, can also be used to accurately evaluate the contribution of BCRP to in vitro drug permeability. In addition, the present method is capable of determining the role of not only BCRP but also P-gp in drug transport. Therefore, we consider that our present method using $A Q$ provides a useful in vitro approach to predict the contribution of BCRP and P-gp to drug absorption.

In conclusion, we summarized the relationship between $P_{\mathrm{AB}}$ and $A Q_{\mathrm{BCRP}}$ of the compounds used in the present study (Fig. 7). The compounds, which are known substrates for BCRP, showed various $P_{\mathrm{AB}}$ and $A Q_{\mathrm{BCRP}}$ values. In particular, the higher contribution of BCRP to the permeability of compounds was observed when the $P_{\mathrm{AB}}$ value was less than $1.0 \times 10^{-6} \mathrm{~cm} / \mathrm{s}$. Regarding the transport of E3S and ciprofloxacin, which have relatively high $P_{\mathrm{AB}}$ values, we suggest that influx transporters, such as OATPs, cooperatively function with BCRP. For these substrates whose transport involves more than two transporters, it is difficult to determine the extent of the contribution of each transporter in an overexpression study. In contrast, Caco- 2 cell monolayers would enable the accurate estimation of the contribution of respective transporters in intestinal permeability. In addition, with respect to drug-drug interactions (DDIs), the Food and Drug Administration released a draft guidance for the industry in 2017, which describes the importance of determining whether the investigated drug is a substrate of P-gp and BCRP to evaluate its DDI potential. Thus, evaluating the P-gp- and BCRP-mediated drug interactions is a key process for drug development, and our present approach using Caco-2 cell monolayers may also be useful to predict DDIs.

\section{Acknowledgments}

We would like to thank Dr. Kenji Kusumoto, Ikuo Nomura, Kohei Shinozaki, and Naoki Shiota (Kyoto Pharmaceutical University) for their technical assistance. We thank Melissa Crawford from Edanz Group (https://en-authorservices.edanzgroup.com/) for editing a draft of this manuscript.

\section{Authorship Contributions}

Participated in research design: Kawahara, Yamamoto, Fujita.
Conducted experiments: Kawahara, Nishikawa, Fujita.

Contributed reagents or analytic tools: Nishikawa, Kono, Fujita.

Performed data analysis: Kawahara, Nishikawa, Fujita.

Wrote or contributed to the writing of the manuscript: Kawahara, Kono, Fujita.

\section{References}

Allikmets R, Schriml LM, Hutchinson A, Romano-Spica V, and Dean M (1998) A human placenta-specific ATP-binding cassette gene (ABCP) on chromosome $4 \mathrm{q} 22$ that is involved in multidrug resistance. Cancer Res 58:5337-5339.

Álvarez AI, Vallejo F, Barrera B, Merino G, Prieto JG, Tomás-Barberán F, and Espín JC (2011) Bioavailability of the glucuronide and sulfate conjugates of genistein and daidzein in breast cancer resistance protein 1 knockout mice. Drug Metab Dispos 39:2008-2012.

Artursson P and Karlsson J (1991) Correlation between oral drug absorption in humans and apparent drug permeability coefficients in human intestinal epithelial (Caco-2) cells. Biochem Biophys Res Commun 175 (3):880-885.

B Shekhawat P and B Pokharkar V (2017) Understanding peroral absorption: regulatory aspects and contemporary approaches to tackling solubility and permeability hurdles. Acta Pharm Sin B 7:260-280.

Dahan A and Amidon GL (2009) Small intestinal efflux mediated by MRP2 and BCRP shifts sulfasalazine intestinal permeability from high to low, enabling its colonic targeting. Am J Physiol Gastrointest Liver Physiol 297:G371-G377.

Dantzig AH and Bergin L (1990) Uptake of the cephalosporin, cephalexin, by a dipeptide transport carrier in the human intestinal cell line, Caco-2. Biochim Biophys Acta 1027:211-217.

Dautrey S, Felice K, Petiet A, Lacour B, Carbon C, and Farinotti R (1999) Active intestinal elimination of ciprofloxacin in rats: modulation by different substrates. $\mathrm{Br} J$ Pharmacol 127: $1728-1734$.

Doyle LA, Yang W, Abruzzo LV, Krogmann T, Gao Y, Rishi AK, and Ross DD (1998) A multidrug resistance transporter from human MCF-7 breast cancer cells. Proc Natl Acad Sci USA 95: $15665-15670$

Drusano GL, Standiford HC, Plaisance K, Forrest A, Leslie J, and Caldwell J (1986) Absolute oral bioavailability of ciprofloxacin. Antimicrob Agents Chemother 30:444-446.

Enokizono J, Kusuhara H, and Sugiyama Y (2007) Effect of breast cancer resistance protein (Bcrp/ Abcg2) on the disposition of phytoestrogens. Mol Pharmacol 72:967-975.

Fagerholm U (2007) Prediction of human pharmacokinetics--gastrointestinal absorption. J Pharm Pharmacol 59:905-916.

Fisher JM, Wrighton SA, Calamia JC, Shen DD, Kunze KL, and Thummel KE (1999) Midazolam metabolism by modified Caco-2 monolayers: effects of extracellular protein binding. J Pharmacol Exp Ther 289:1143-1150.

Frølund S, Marquez OC, Larsen M, Brodin B, and Nielsen CU (2010) $\delta$-aminolevulinic acid is a substrate for the amino acid transporter SLC36A1 (hPAT1). British Journal of Pharmacology 159:1339-1353.

Ge S, Wei Y, Yin T, Xu B, Gao S, and Hu M (2017) Transport-glucuronidation classification system and PBPK modeling: new approach to predict the impact of transporters on disposition of glucuronides. Mol Pharm 14:2884-2898.

Gram LK, Rist GM, Lennernäs H, and Steffansen B (2009) Impact of carriers in oral absorption: permeation across Caco-2 cells for the organic anions estrone-3-sulfate and glipizide. Eur J Pharm Sci 37:378-386.

Grube M, Reuther S, Meyer Zu Schwabedissen H, Köck K, Draber K, Ritter CA, Fusch C, Jedlitschky G, and Kroemer HK (2007) Organic anion transporting polypeptide 2B1 and breast cancer resistance protein interact in the transepithelial transport of steroid sulfates in human placenta. Drug Metab Dispos 35:30-35.

Haslam IS, Wright JA, O'Reilly DA, Sherlock DJ, Coleman T, and Simmons NL (2011) Intestinal ciprofloxacin efflux: the role of breast cancer resistance protein (ABCG2). Drug Metab Dispos 39:2321-2328.

Hilgendorf C, Ahlin G, Seithel A, Artursson P, Ungell A-L, and Karlsson J (2007) Expression of thirty-six drug transporter genes in human intestine, liver, kidney, and organotypic cell lines. Drug Metab Dispos 35:1333-1340.

Jambhekar SS and Breen PJ (2013) Drug dissolution: significance of physicochemical properties and physiological conditions. Drug Discov Today 18:1173-1184.

Jekerle V, Klinkhammer W, Reilly RM, Piquette-Miller M, and Wiese M (2007) Novel tetrahydroisoquinolin-ethyl-phenylamine based multidrug resistance inhibitors with broad-spectrum modulating properties. Cancer Chemother Pharmacol 59:61-69.

Jekerle V, Klinkhammer W, Scollard DA, Breitbach K, Reilly RM, Piquette-Miller M, and Wiese M (2006) In vitro and in vivo evaluation of WK-X-34, a novel inhibitor of P-glycoprotein and $\mathrm{BCRP}$, using radio imaging techniques. Int J Cancer 119:414-422.

Kage K, Tsukahara S, Sugiyama T, Asada S, Ishikawa E, Tsuruo T, and Sugimoto Y (2002) Dominant-negative inhibition of breast cancer resistance protein as drug efflux pump through the inhibition of S-S dependent homodimerization. Int J Cancer 97:626-630.

Keldenich J (2009) Measurement and prediction of oral absorption. Chem Biodivers 6:2000-2013. Kobayashi S, Shinohara M, Nagai T, and Konishi Y (2013) Transport mechanisms for soy isoflavones and microbial metabolites dihydrogenistein and dihydrodaidzein across monolayers and membranes. Biosci Biotechnol Biochem 77:2210-2217.

Kono Y, Jinzai H, Kotera Y, and Fujita T (2017) Influence of Physicochemical Properties and PEG Modification of Magnetic Liposomes on Their Interaction with Intestinal Epithelial Caco-2 Cells. Biol Pharm Bull 40:2166-2174.

Kruijtzer CMF, Beijnen JH, Rosing H, ten Bokkel Huinink WW, Schot M, Jewell RC, Paul EM, and Schellens JHM (2002) Increased oral bioavailability of topotecan in combination with the breast cancer resistance protein and P-glycoprotein inhibitor GF120918. J Clin Oncol 20: 2943-2950.

Kusuhara H, Furuie H, Inano A, Sunagawa A, Yamada S, Wu C, Fukizawa S, Morimoto N, Ieiri I, Morishita M, et al. (2012) Pharmacokinetic interaction study of sulphasalazine in healthy subjects and the impact of curcumin as an in vivo inhibitor of BCRP. $B r J$ Pharmacol 166: 1793-1803.

Larregieu CA and Benet LZ (2013) Drug discovery and regulatory considerations for improving in silico and in vitro predictions that use Caco-2 as a surrogate for human intestinal permeability measurements. AAPS J 15 (2):483-497. 
Lipinski CA (2000) Drug-like properties and the causes of poor solubility and poor permeability. J Pharmacol Toxicol Methods 44:235-249.

Lu X, Chan T, Zhu L, Bao X, Velkov T, Zhou QT, Li J, Chan HK, and Zhou F (2018) The inhibitory effects of eighteen front-line antibiotics on the substrate uptake mediated by human organic anion/cation transporters, organic anion transporting polypeptides and oligopeptide transporters in in vitro models. Eur J Pharm Sci 115:132-143.

Maeda T, Takahashi K, Ohtsu N, Oguma T, Ohnishi T, Atsumi R, and Tamai I (2007) Identification of influx transporter for the quinolone antibacterial agent levofloxacin. Mol Pharm 4: $85-94$.

Maliepaard M, Scheffer GL, Faneyte IF, van Gastelen MA, Pijnenborg AC, Schinkel AH, van De Vijver MJ, Scheper RJ, and Schellens JH (2001) Subcellular localization and distribution of the breast cancer resistance protein transporter in normal human tissues. Cancer Res 61:3458-3464.

Mao Q and Unadkat JD (2005) Role of the breast cancer resistance protein (ABCG2) in drug transport. AAPS J 7:E118-E133.

Matsuda Y, Konno Y, Hashimoto T, Nagai M, Taguchi T, Satsukawa M, and Yamashita S (2013) In vivo assessment of the impact of efflux transporter on oral drug absorption using portal veincannulated rats. Drug Metab Dispos 41:1514-1521.

Merino G, Alvarez AI, Pulido MM, Molina AJ, Schinkel AH, and Prieto JG (2006) Breast cancer resistance protein (BCRP/ABCG2) transports fluoroquinolone antibiotics and affects their oral availability, pharmacokinetics, and milk secretion. Drug Metab Dispos 34:690-695.

Merino G, Jonker JW, Wagenaar E, van Herwaarden AE, and Schinkel AH (2005) The breast cancer resistance protein (BCRP/ABCG2) affects pharmacokinetics, hepatobiliary excretion, and milk secretion of the antibiotic nitrofurantoin. Mol Pharmacol 67:1758-1764.

Miyake K, Mickley L, Litman T, Zhan Z, Robey R, Cristensen B, Brangi M, Greenberger L, Dean M, Fojo T, et al. (1999) Molecular cloning of cDNAs which are highly overexpressed in mitoxantroneresistant cells: demonstration of homology to ABC transport genes. Cancer Res 59:8-13.

Nielsen CU, Carstensen M, and Brodin B (2012) Carrier-mediated $\gamma$-aminobutyric acid transport across the basolateral membrane of human intestinal Caco-2 cell monolayers. Eur J Pharm Biopharm 81:458-462.

Ramanathan S, Pooyan S, Stein S, Prasad PD, Wang J, Leibowitz MJ, Ganapathy V, and Sinko PJ (2001) Targeting the sodium-dependent multivitamin transporter (SMVT) for improving the oral absorption properties of a retro-inverso Tat nonapeptide. Pharm Res 18:950-956.

Sai Y, Kaneko Y, Ito S, Mitsuoka K, Kato Y, Tamai I, Artursson P, and Tsuji A (2006) Predominant contribution of organic anion transporting polypeptide OATP-B (OATP2B1) to apical uptake of estrone-3-sulfate by human intestinal Caco-2 cells. Drug Metab Dispos 34:1423-1431.

Siissalo S, Zhang H, Stilgenbauer E, Kaukonen AM, Hirvonen J, and Finel M (2008) The expression of most UDP-glucuronosyltransferases (UGTs) is increased significantly during Caco-2 cell differentiation, whereas UGT1A6 is highly expressed also in undifferentiated cells. Drug Metab Dispos 36:2331-2336.
Suzuki M, Suzuki H, Sugimoto Y, and Sugiyama Y (2003) ABCG2 transports sulfated conjugates of steroids and xenobiotics. $J$ Biol Chem 278:22644-22649.

Tachibana T, Kitamura S, Kato M, Mitsui T, Shirasaka Y, Yamashita S, and Sugiyama Y (2010) Model analysis of the concentration-dependent permeability of P-gp substrates. Pharm Res 27: 442-446.

Taipalensuu J, Törnblom H, Lindberg G, Einarsson C, Sjöqvist F, Melhus H, Garberg P, Sjöström B, Lundgren B, and Artursson P (2001) Correlation of gene expression of ten drug efflux proteins of the ATP-binding cassette transporter family in normal human jejunum and in human intestinal epithelial Caco-2 cell monolayers. J Pharmacol Exp Ther 299:164-170.

Takada T, Suzuki H, and Sugiyama Y (2005) Characterization of polarized expression of point- or deletion-mutated human BCRP/ABCG2 in LLC-PK1 cells. Pharm Res 22:458-464.

Thwaites DT, Hirst BH, and Simmons NL (1994) Substrate specificity of the di/tripeptide transporter in human intestinal epithelia (Caco-2): identification of substrates that undergo $\mathrm{H}^{+}$. coupled absorption. Br J Pharmacol 113:1050-1056.

Troutman MD and Thakker DR (2003) Novel experimental parameters to quantify the modulation of absorptive and secretory transport of compounds by P-glycoprotein in cell culture models of intestinal epithelium. Pharm Res 20:1210-1224.

Voigt V, Laug L, Zebisch K, Thondorf I, Markwardt F, and Brandsch M (2013) Transport of the areca nut alkaloid arecaidine by the human proton-coupled amino acid transporter 1 (hPAT1) $J$ Pharm Pharmacol 65:582-590.

Volpe DA (2008) Variability in Caco-2 and MDCK cell-based intestinal permeability assays. J Pharm Sci 97:712-725.

Wang H, Dutta B, Huang W, Devoe LD, Leibach FH, and Ganapathy V (1999) Human $\mathrm{Na}^{+}$. dependent vitamin $\mathrm{C}$ transporter 1 (hSVCT1): primary structure, functional characteristics and evidence for a non-functional splice variant. Biochim Biophys Acta 1461:1-9.

Wright JA, Haslam IS, Coleman T, and Simmons NL (2011) Breast cancer resistance protein BCRP (ABCG2)-mediated transepithelial nitrofurantoin secretion and its regulation in human intestinal epithelial (Caco-2) layers. Eur J Pharmacol 672:70-76.

Xiao Y, Davidson R, Smith A, Pereira D, Zhao S, Soglia J, Gebhard D, de Morais S, and Duignan DB (2006) A 96-well efflux assay to identify ABCG2 substrates using a stably transfected MDCK II cell line. Mol Pharm 3:45-54.

Address correspondence to: Dr. Takuya Fujita, Laboratory of Molecular Pharmacokinetics, Graduate School of Pharmaceutical Sciences, Ritsumeikan University, 1-1-1 Nojihigashi, Kusatsu, Shiga 52-8577, Japan. E-mail: fujita-t@ph. ritsumei.ac.jp 